\title{
Tubercular Mastitis in an Elderly Female: A Rare Case Report
}

\section{Manvendu Jha ${ }^{1}$, Hakam Singh ${ }^{2}$ and Amulyajeet Kaur ${ }^{1}$}

${ }^{1}$ Military hospital Srinagar, India

${ }^{2}$ Military hospital Chandigarh, India

\section{ABSTRACT}

Introduction: Tuberculosis of the breast is a rare entity, especially in elderly females. Moreover, the disease is overlooked and misdiagnosed as malignancy or pyogenic abscess. Here we report a case of an elderly female who presented with a lump in her left breast which resembled malignancy. Fine needle aspiration cytology followed by histopathological examination confirmed the diagnosis of breast tuberculosis. Patient underwent excision of the lump followed by six months of anti tubercular therapy to which she responded well.

Key words: breast; mastitis; tuberculosis
\end{abstract}

Correspondence: Manvendu Jha, Military Hospital, Srinagar, India. E-mail: jhamanvendu@rediffmail.com DOI: $10.3126 / \mathrm{mjsbh} . v 20 \mathrm{i} 2.33226$

Submitted on: $2020-12-05$

Accepted on: 2021-06-08 


\section{INTRODUCTION}

Tuberculous mastitis is a rare presentation of tuberculosis that has been called the 'great masquerader' due to its multifaceted presentations. Solitary breast mass is the commonest clinical presentation of tuberculous mastitis and is associated with inflammatory findings in the majority of cases. It is an uncommon disease even in countries with a high incidence of pulmonary and extrapulmonary tuberculosis. The diagnosis of breast tuberculosis usually remains obscure and a multidisciplinary team approach is needed to establish a timely and correct diagnosis. ${ }^{1}$

\section{CASE REPORT}

Sixty three years old, post-menopausal female with no family history of breast cancer reported with a lump in her left breast of eight months duration. There was no history of any increase in size of the lump, any nipple discharge or sinus, fever or weight loss. There was no history of any previous breast surgery or exposure to a patient of tuberculosis. There was no history of any lump in the axilla. Physical examination revealed the patient to be of average built and nourishment. A $3 \times 3 \mathrm{~cm}$ lump was palpable in the upper inner quadrant of left breast, which was non tender, firm to hard in consistency, fixed to the overlying skin but mobile over underlying breast tissue. Skin over breast and nipple areola complex was normal and there was no axillary or supraclavicular lymphadenopathy. Haematological and biochemical

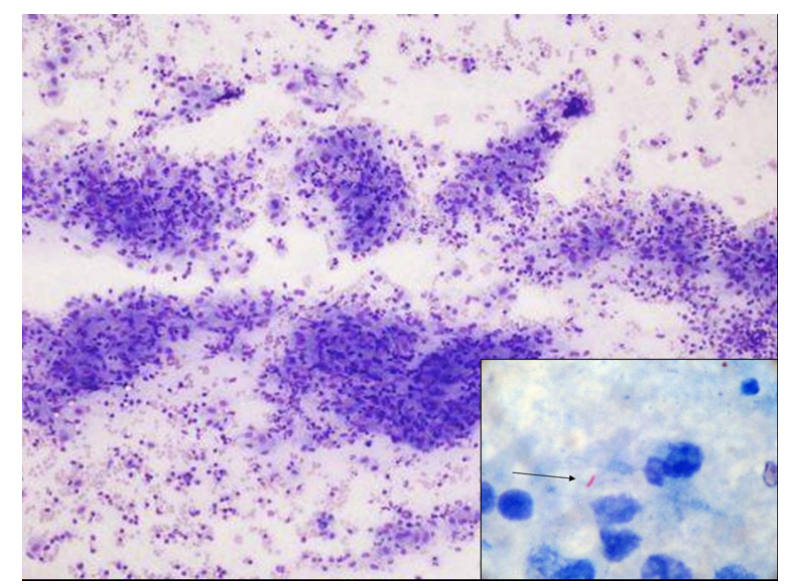

Figure 1. Photomicrograph of fine needle aspirate smear showing epithelioid cell granulomas and acid fast bacilli (inset) on ZN stain. Leishman Giemsa, x 400 (inset) ZN stain, x 1000 parameters were normal. Mammography (Figure 1) revealed a poorly marginated lesion with spiculated margins in the upper inner quadrant of left breast with distortion of surrounding architecture; highly suggestive of malignancy- BIRAD'S-5. During FNAC, $3 \mathrm{ml}$ of thick pus was aspirated. FNAC smears (Figure 2) revealed necrotic debris with scattered epitheloid granulomas and multinucleate giant cells. Neutrophils, plasma cells and lymphocytes were present in the background. AFB stain was positive for acid fast bacilli. However, culture for AFB was negative. In view of the above findings, a diagnosis of mycobacterial mastitis was made. However, taking into consideration patient's age and mammographic findings, wide local excision of the lump was done. Histo-pathological examination confirmed the diagnosis of tuberculosis. She was administered six months of anti tubercular therapy. Patient was followed up for one year. Her surgical wound healed well. There was no pain or recurrence of lump.

\section{DISCUSSION}

Mycobacterial mastitis is a rare form of extra pulmonary tuberculosis despite one third of population being infected with tubercle bacilli. First case of mammary tuberculosis was reported by Sir Ashley Cooper in 1829 who called it 'Scrofulous swelling of the bossom.' ${ }^{2}$ Breast tuberculosis commonly affects women in the reproductive age group, between 21 to 30 years. It most commonly presents as a lump in the central or upper quadrant

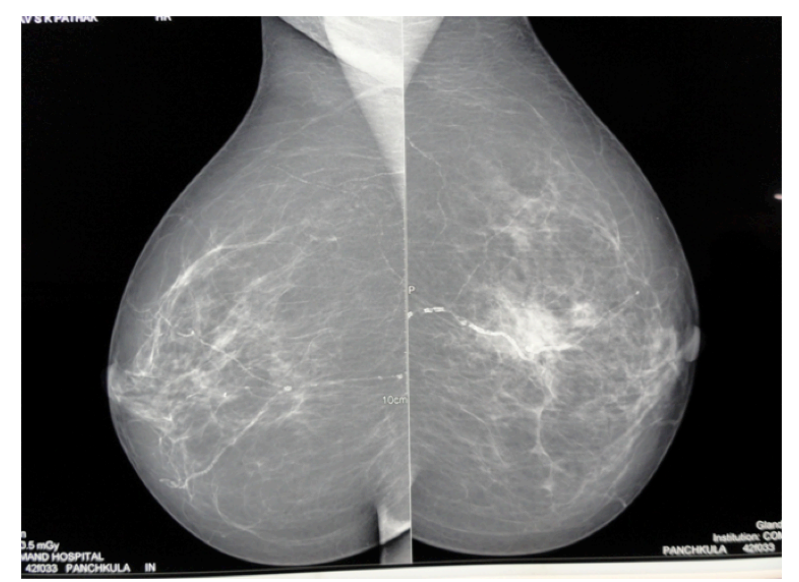

Figure 2. Mammography showing a poorly marginated lesion with spiculated margins in the upper inner quadrant of left breast with distortion of surrounding architecture; highly suggestive of malignancy. BIRAD'S-5 
of the breast. It can also present as oedema of the breast or as a breast abscess.

The history of presenting symptoms in breast tuberculosis is usually less than a year but varies from a few months to several years. ${ }^{3}$ Breast tuberculosis commonly affects women in their reproductive age group, between 21 to 30 years. It is uncommon in prepubescent females and elderly women as in our case. Bilateral involvement is uncommon (3\%). It most commonly presents as a lump ${ }^{4}$ in the central and upper outer quadrant of the breast. In our patient, the lump was present in the upper inner quadrant. Tubercular mastitis is probably due to extension of tubercular infection from axillary nodes to the breast, but the lump is usually painful which was not so in our case. Breast remains mobile unless involvement is there in the chest wall. Tubercular ulcer of breast skin and tubercular breast abscess with or without discharging sinuses are other forms of presentation. ${ }^{5}$ Peau d' orange is often seen in patients with extensive axillarynodal tuberculosis. Purulent nipple discharge or persistent discharging sinus may be the rare presenting feature. Our patient did not have any such features.

Diagnosis warrants a high index of suspicion on clinical, pathological or microbiological confirmation of all suspected lesions. Mantoux's test is usually positive in adults in endemic areas of tuberculosis and is not of great help in diagnosis. Fine needle aspiration cytology (FNAC) from the breast lesion continues to remain an important diagnostic tool of breast tuberculosis as in our patient. Approximately $73 \%$ cases of breast tuberculosis can be diagnosed on FNAC when both epitheloid cell granulomas and necrosis are present. The demonstration of acid-fastbacilli (AFB) on FNAC is not mandatory, but in our case we were able to demonstrate AFB. Though mycobacterial culture remains the gold standard for diagnosis of tuberculosis, frequent negative results (as in our case) in paucibacillary specimens are important limitations. However, open biopsy of breast lump, ulcer, sinus or wall of a suspected tubercular breast abscess cavity almost always confirms breast tuberculosis. ${ }^{6}$ Wide local excision of the lump, not only relieved the patient of presenting complaint it also helped in clinching the diagnosis. Polymerase chain reaction (PCR) in the diagnosis of breast tuberculosis is less often reported, mostly as a tool to distinguish tubercular mastitis from other forms of granulomatous mastitis. However, PCR is by no means absolute in diagnosing tubercular infection and false negative report is still a possibility. ${ }^{7} \mathrm{We}$ did not do this investigation in our patient.

Duration of antitubercular therapy as documented in literature has been six months (Two months intensive + four months continuation phase), extending to nine months (Two months intensive + seven months continuation) in some unresponsive cases. ${ }^{8,9}$ However, consensus has been six months of therapy, which we also followed in our case, with successful outcome.

\section{CONCLUSIONS}

Tubercular mastitis is a rare even in endemic population. Its presence in an elderly patient with a breast lump and without the tell-tale signs of chronic non healing ulcer / sinus makes it difficult to diagnose and requires a multidisciplinary approach. Hence in evaluation of any breast lump across the age groups, it should always be kept in the differential diagnosis, especially in tubercular epidemic regions of the world like ours.

To cite this article: Jha M, Singh H, Kaur A. Tubercular Mastitis in an Elderly Female: A Rare Case Report. MJSBH. 2021;20(2):177-80.

Conflict of Interest: None declared 


\section{REFERENCES}

1. Farrokh D, Alamdaran A, Ali FL, Yalda FR, Bita A. Tubercular Mastitis. A review of 32 cases. Int J Infectious diseases 2019;87: 135-42. DOI: 10.1016/j.ijid.2019.08.0.013

2. Kakkar S, Kapila K, Singh MK, Verma K. Tuberculosis of breast: a cytomorphologic study. Acta cytol 2000; 44: 292-96. DOI: 10.1159/000328467

3. Dubey MM, Agarwal S. Tuberculosis of the breast. J Indian Med Assoc 1968;51:358-9. PMID: 5705887

4. Alagratnam TT, Ong GB. Tuberculosis of the breast. Br J Surg. 1980;67:125-6. DOI: 10.1002/bjs. 1800670216.

5. Shukla HS, Kumar S. Benign breast disorders in non-western population: Part II-Benign breast disorders in India. World J Surg. 1995;13:746-9. DOI: 10.1007/BF01658426

6. Shinde SR, Chandrawar RY, Deshmukh SP. Tuberculosis of the breast masquerading a carcinoma: a study of 100 patients. World J Surg. 1995;19:379-8. DOI: 10.1007/BF00299163.

7. Katoch VM. New diagnostic techniques for tuberculosis. Indian J Med Res. 2004;120:418-28 . PMID: 15520490

8. S Baharoon. Tuberculosis of the breast. Ann Thorac Med. 2008;3:110-14. DOI: $10.4103 / 1817-1737.41918$

9. Jalali U, Rasul S, Khan A, Baig N, Akhter R. Tubercular Mastitis. J Coll Physicians Surg Pak. 2005;15:234-37. DOI: 04.2005/jcpsp.234237 